\title{
Obtención de una Denominación de Origen Protegido ANIMAL EN ECUADOR EN BASE A LA NORMATIVA EUROPEA
}

\section{ANIMAL PROTECTED DESIGNATION OF ORIGEN AT ECUADOR ACCORDING TO}

\author{
UE NORMATIVE
}

\author{
Manuel Esteban Maldonado Cornejo ${ }^{1, *}$, Ramón Casals Costa ${ }^{2}$, \\ Xavier Such Martí ${ }^{2}$, María Cristina Narváez Riofrío ${ }^{3}$
}

\footnotetext{
1* Universidad Católica de Cuenca, Agropampilla, Av. de las Américas y Humboldt, Cuenca, Ecuador.

${ }^{2}$ Universidad Autónoma de Barcelona, Plaça Cívica, 08193 Bellaterra, Barcelona, España.

${ }^{3}$ Instituto Nacional de Investigación en Salud Pública Dr. Leopoldo Izquieta Pérez, Av. Julián Coronel 905 entre Esmeraldas y José Mascote Av. Juan Tanca Marengo No. 100 y Av. de las Américas Guayaquil, Ecuador.
}

*Autor para correspondencia: mmaldonadoc@ucacue.edu.ec, mmaldonado@pampilla.com

\begin{abstract}
Resumen
La poca adaptabilidad de los productos tradicionales frente a la globalización hace que pierdan mercado. Dado que una de las principales limitaciones para estos productos es su comercialización bajo estándares mínimos de calidad, surge la pregunta ¿Es posible obtener productos tradicionales con una calidad reconocible? En la UE la respuesta a esta pregunta se encuentra básicamente en el marco de las Denominaciones de Origen Protegidas (DOP). Una DOP otorga un valor agregado al producto bajo un concepto general de calidad, definida por su propia identidad, permitiéndole aproximarse a los estándares esperados por el consumidor. El principal objetivo de este trabajo es desarrollar un ejercicio para una propuesta local para la obtención de una DOP en Ecuador, estudiando previamente los principios generales para una denominación de origen ecuatoriana, comparándolos con la visión europea de una DOP. Para ellos se ha definido el alcance general de su aplicación y se ha estudiado el marco legal para su implementación. Por último, se ha desarrollado un pliego de condiciones con base en el "Queso de Hoja de Cayambe", que podría servir como referencia para la implementación de una DOP en cualquier otro producto. Concluyendo que para el funcionamiento de una DOP en el Ecuador, es necesario encontrar flexibilidad administrativa, voluntad política y participación científica; pudiéndose usar este trabajo como herramienta de partida para el desarrollo de proyectos comunitarios agropecuarios y alimentarios.
\end{abstract}

Palabras clave: Calidad, DOP, identidad, Ecuador, UE. 


\begin{abstract}
Amid globalization causes traditional products with low adaptability to lose market share. Considering that one of the main limitation of traditional products is their marketability under minimum quality standards, the following question arises: Is it possible to obtain traditional products with a level of quality recognizable by consumers? In the EU the answer for this question is found mainly in context of the protected designations of origin (PDO). A PDO status adds quality to the product by the value of its own identity, reflecting the quality standards expected by the consumer. The main objective of this work is to develop a proposal to obtain a PDO in Ecuador, studying the general principles of a designation of origin in Ecuador and comparing them with the European vision of a PDO of animal origin. Therefore, this paper defines the commercial, social, and agricultural aspects of the implementation of a PDO. Using an inter-regional approach, several products eligible for carrying the PDO seal are compared, and the legal framework for implementation in Ecuador is considered. Finally, a list of conditions based on the "Queso de Hoja de Cayambe" (Cayambe "string" cheese) has been developed, which could be used as a benchmark for implementation of a PDO on other products. The conclusion is that to implement a PDO in Ecuador, administrative flexibility, political will, and scientific engagement are needed. This study may be used as a preliminary tool for the development of community projects focused on livestock agriculture and food products.
\end{abstract}

Keywords: Quality, PDO, identity, Ecuador, EU.

Forma sugerida de citar: Maldonado Cornejo, M. E., Casals Costa, R., Such Martí, X., Narváez Riofrío, M. C.. 2018. Obtención de una Denominación de Origen Protegido de origen animal en Ecuador en base a la normativa europea. La Granja: Revista de Ciencias de la Vida. Vol. 27(1):6-20. http://doi.org/10.17163/lgr.n27.2018.01.

Parte de la presente investigación se presentó en la Coordinación General del Sistema de Información del Ministerio de Agricultura, Ganadería Acuacultura y Pesca del Ecuador existe un Propuesta para el "Estudio comparativo para obtención de una D.O.P. en Ecuador en base al caso europeo" (Maldonado, 2017). 


\section{Introducción}

Los estudios comparativos entre distintas regiones en relación con el fomento de procesos productivos son una herramienta clave para entender cualquier propuesta que se realice para su puesta en marcha y funcionamiento. Dado que las comunidades campesinas latinoamericanas representan un núcleo de población muy sensible frente a la globalización, y que las políticas comerciales no les permiten en muchos casos lograr un desarrollo socioeconómico inclusivo para todos los participantes (Vandecandelaere et al., 2010), implementar una Denominación de Origen Protegido (DOP) puede constituir una gran oportunidad para aprovechar las capacidades productivas de los sectores rurales latinoamericanos en pos del comercio justo (Tolentino Martínez, 2015), promoviendo la organización social y el fomento de proyectos comunitarios (Unión Europea, 2015).

En el Ecuador, en el literal 2 del Artículo 385, Sección Octava de Ciencia, Tecnología y saberes Ancestrales, Capitulo Primero sobre la Inclusión y Equidad del Título VII del Régimen del Buen Vivir indica que "el sistema nacional de ciencia, tecnología, innovación y saberes ancestrales, en el marco del respeto al ambiente, la naturaleza, la vida, las culturas y la soberanía, tendrá como finalidad el recuperar, fortalecer y potenciar los saberes ancestrales, desarrollando tecnologías e innovaciones que impulsen la producción nacional, eleven la eficiencia y productividad" (Asamblea Constituyente, 2008), enfocado posteriormente en los principios, del Código Orgánico de Economía Social del Conocimiento e Innovación (Asamblea Nacional, 2016), como iniciativa estatal para la protección de las tradiciones, en el Ecuador.

El presente trabajo pretende desarrollar una propuesta para la obtención de una DOP en Ecuador teniendo en consideración la normativa ecuatoriana y la europea, a partir de: 1) Revisar la definición y bases para el desarrollo de una DOP en Europa, vinculando estos principios con la calidad de alimentos de origen animal. 2) Identificar un producto local y desarrollar un pliego de condiciones para el establecimiento de una DOP de origen animal.

\section{Definición}

Un producto con un sello de indicación geográfica es aquél cuyo origen tradicional y características es- tán relacionadas con una zona geográfica específica. Estas características cualitativas deben ser comprobables y vinculadas con esta localidad. En Europa se ha hecho distinción entre estos alimentos y dependiendo de sus características se los ha dividido en tres categorías: Denominación de Origen Protegido (DOP), Indicación Geográfica Protegida (IGP) y Especialidad Gastronómica Protegida (EGT). En el Ecuador, y debido a que la Ley no distingue entre denominaciones de origen e indicaciones geográficas, en el presente trabajo se hace mención de forma general a estos productos como una DOP.

Así, una DOP se definiría, de forma general, como el sello que se da a un producto tradicional, consumido con frecuencia elaboración ha sido trasmitida históricamente a través de generaciones, y sus características sensoriales están ligadas a su origen geográfico (Guerrero et al., 2010).

\section{Alcances}

Las DOP están siempre asociadas a una protección comercial para los pequeños y medianos productores, y han sido enfocadas en mejorar su remuneración económica (Consejo de la Unión Europea, 2012). El consumidor asocia el sello como un símbolo de calidad, y dado que la calidad es rentable, el producto se relacionará con los intereses del consumidor (Sellers y Nicolau, 2001). El consumidor europeo es cada vez más crítico respecto a los alimentos de origen animal, por lo que suele buscar en ellos su procedencia y sostenibilidad en la elaboración, mientras que los alimentos tradicionales en el Ecuador tienen otras características, como se muestra en la Tabla 1. Por lo que los alcances de una DOP son diversos, y pueden ir desde la parte comercial hasta un posible enfoque agro-sostenible.

En el Ecuador, pocas comunidades sienten la necesidad de asociarse como una alternativa al fortalecimiento de su producción (Hentschel y Waters, 2002) por lo que una DOP puede ser una alternativa para fomentar la asociación productiva. Para desarrollar una DOP es importante establecer la cadena de valor de los productos que identifican a un producto con la sociedad en la que se produce, para que posteriormente la sociedad lo califique de acuerdo con sus normas civiles y su entorno (Vandecandelaere et al., 2010). Una DOP busca el desarrollo de una sociedad, y constituye una herramienta muy útil para su desarrollo. En especial promueve la protección de grupos vulnerables (Oyarzun, 2011). 
Tabla 1. Comparativa entre Europa y Ecuador de las características de los alimentos aptos para una DOP (Unión Europea, 2014; Guardia, 2016; IEPI, 2016).

\begin{tabular}{|c|c|}
\hline Europa & Ecuador \\
\hline \multicolumn{2}{|l|}{ 1. Alimentos seguros } \\
\hline 2. Respeta la demanda del consumidor & $\begin{array}{l}\text { 1. Calidad Higiénica Diversa } \\
\text { 2. Alta aceptación }\end{array}$ \\
\hline $\begin{array}{l}\text { 3. Oferta limitada por estacionalidad o } \\
\text { limitación de zona de producción }\end{array}$ & $\begin{array}{l}\text { 3. Oferta limitada por el tamaño de zo- } \\
\text { na de producción }\end{array}$ \\
\hline $\begin{array}{l}\text { 4. Reconocidos por su responsabilidad } \\
\text { social }\end{array}$ & $\begin{array}{l}\text { 4. Reconocidos por su carácter comuni- } \\
\text { tario }\end{array}$ \\
\hline 5. De diferentes formas de producción & $\begin{array}{l}\text { 5. Asociados a procesos de producción } \\
\text { Agroecológicos }\end{array}$ \\
\hline
\end{tabular}

\section{Características de los Productos Aptos Para una DOP}

Un producto será apto para ser considerado una DOP según sus características de origen y el vínculo del mismo con el área geográfica, y en el caso de los alimentos tradicionales si su permanencia en el mercado local le ha permitido la transmisión de sus características a lo largo de distintas generaciones de productores y consumidores (Consejo de la Unión Europea, 2012).

La Unión Europea considera aptos para una DOP de origen animal todos los productos especificados en el Anexo I del Reglamento (CE) 510/2006 (Consejo de la Unión Europea, 2006), entre los que se encuentran: carnes frescas, despojos cárnicos comestibles (tripas, vísceras, estómagos), pescados, preparados de carne, pescado, crustáceos o moluscos, mantecas animales, quesos, productos lácteos, huevos y mieles.

En el Ecuador el tema es más genérico, y se considera apto para ser considerado DOP cualquier producto de origen agroindustrial que cumpla con las características básicas de una denominación de origen (IEPI, 2016).

La identificación de estos productos varía entre estas dos regiones. Mientras en Europa se usa un sello fácilmente reconocido por el consumidor, en Ecuador los productos tradicionales necesitan reconocimiento del estado según se muestra en la Tabla 2 .

Tabla 2. Comparativo de Sellos de Reconocimiento de Productos Tradicionales entre Europa y Ecuador (Unión Europea, 2014; IEPI, 2016).

\begin{tabular}{cc|}
\hline Europa & Ecuador \\
\hline Sello reconocido por el consumidor & Necesita reconocimiento del Estado \\
\hline
\end{tabular}

En Ecuador se pueden identificar varios sistemas de producción agropecuarios que se adaptan específicamente a su medio geográfico particular. A la vez, se han identificado varios productos potenciales para una DOP de origen animal. Sobre todo, para poder obtenerlos, es necesaria una capacitación total del sistema en los procesos de producción agroalimentaria (IEPI, 2016).

En la actualidad existen dos denominaciones reconocidas como son: el "Cacao de Arriba" de origen vegetal y el "Sombrero Montecristi" de características artesanales. Además, se encuentran en proyecto para obtener una DOP el Café Galápagos, otros productos alimentarios y varias artesanías. 


\section{Principios de un producto apto para una DOP}

Para identificar un producto apto para una DOP es necesario contrastar y definir las diferencias del producto con otros similares, según una diferenciación de las características del producto en diferentes regiones (Oberthür et al., 2011).

En la actualidad los productos con DOP son reconocidos a nivel mundial por la OMC, siempre y cuando esta denominación haya sido aceptada en su país de origen y cumpla con las normas del país de destino (WTO, 2012). De esta forma se facilita el comercio internacional de los productos tradicionales y su reconocimiento global.

Desde el 2006 se declaró en la Unión Europea la aceptación de solicitudes de DOP de terceros países (WTO, 2009), y fue el "Café Colombia" el primer producto latinoamericano reconocido en Europa (Café de Colombia, 2009).

La armonización legislativa entre países facilita el intercambio económico, y para que éste se realice de forma segura es necesario garantizar los niveles de calidad de los productos. Es por este motivo que para la obtención de una DOP es necesario el desa- rrollo de un procedimiento legal que reconozca al producto como tal.

\section{Marco legal europeo}

En Europa, un término bajo la inscripción DOP sirve para identificar a un producto que ha cumplido un pliego de condiciones que garantizan su calidad en todas las fases de producción. Este sello es reconocido en toda la Unión Europea como garantía de originalidad y tradición de acuerdo con el Arreglo de Lisboa (Unión de Lisboa, 1979).

En Europa se han desarrollado diversos programas de protección agrícola, como las políticas sobre la estructura agraria que promueven la mejora, adaptación y desarrollo en las zonas rurales. El Reglamento 1151/2012 (Consejo de la Unión Europea, 2012) del Parlamento y Consejo Europeo está enfocado a una amplia gama de productos de este tipo, y tiene como objetivo regular y promover la competencia leal y los derechos de propiedad intelectual. En la Tabla 3 se detalla el procedimiento general para la obtención de una DOP en Europa con base en este reglamento.

Tabla 3. Pasos para la obtención de una DOP en Europa: solicitud, condiciones, inspección y certificación (Consejo de la Unión Europea, 2012)

\begin{tabular}{l} 
1. Solicitud \\
$\begin{array}{l}\text { Petición administrativa del país o región (en nombre de personas físicas, mora- } \\
\text { les, públicas o privadas) según: El Tratado de Lisboa y el Reglamento (Unión de } \\
\text { Lisboa, 1979). }\end{array}$ \\
$\begin{array}{l}\text { 2. Condiciones } \\
\text { Pliego de condiciones justificando: el área geográfica de influencia y las caracte- } \\
\text { rísticas del producto. }\end{array}$ \\
$\begin{array}{l}\text { La Comisión Europea (CE) estudiará el caso, notificará y actuará como árbitro si } \\
\text { el caso lo amerita. }\end{array}$ \\
$\begin{array}{l}\text { El registro de una DOP confiere derechos de comercialización mientras los esta- } \\
\text { dos de la UE velarán por qué estos se cumplan. }\end{array}$ \\
\hline
\end{tabular}

\section{$7 \quad$ Marco legal ecuatoriano}

En Latinoamérica, la diversidad de productos tradicionales coincide con una gran variabilidad legislativa entre países productores (Molina, 2015). Los distintos tratados multilaterales de propiedad intelectual buscan reducir al mínimo el fraude de los productos, siendo la inestabilidad legal una limitante en la promoción de los mismos. Es así que los primeros aportes legislativos en esta línea se pro- 
ducen durante la Ronda de Uruguay de Propiedad Intelectual (ADPIC, 1994). En la actualidad, países como Argentina y Chile han promovido legislaciones específicas para estos casos, como sucede con la Ley 25380/2004 (Senado Argentino, 2004) y la Ley 19039, Título IX (Legislación Chilena, 2005). Por otra parte, los países andinos han planteado de forma más general sus normas. Así lo recogen los artículos 201 a 223 de la decisión 486 sobre la Propiedad Industrial de la Comunidad Andina, que mencionan ciertas bases generales para el manejo de las DOP en la región (Comisión de la Comunidad Andina, 2000).

En el Ecuador la regulación jurídica de las DOP forma parte del Código Orgánico de la Economía Social de los Conocimientos, Creatividad e Inno- vación, Capitulo XII (Asamblea Nacional, 2016) en donde se establece que se designará una Denominación de Origen a un producto de una zona geográfica determinada en la cuál se produce, extrae y elabora el mismo, incluyendo sus factores humanos y naturales. Este código no hace distinción entre DOP e IGP, pero ya incluye a las EGT en otro apartado. Entre lo más destacable del mismo esta la priorización de la producción comunitaria sobre otro tipo de producción y algunas especificaciones sobre su uso y certificación frente a la autoridad competente según se establece en la Tabla 4 . Es por esto que al otorgarles una DOP a estos productos se logra su protección, ya que se transforman en "un bien de la nación confiado a sus productores" (Llorente, 2001).

Tabla 4. Pasos Para la Obtención de una D.O.P. en Ecuador: Solicitud, Condiciones, Inspección y Certificación. (IEPI, 2016)

\section{Solicitud}

Petición de persona natural o jurídica dedicada a la producción y/o elaboración del producto, autorizada como representante legal de la Indicación Geográfica según: La Dirección Nacional de Propiedad Intelectual (IEPI).

\section{Condiciones}

Especificar: Área geográfica de influencia y las características del producto.

\section{Inspección}

El IEPI estudiará el caso e inspeccionará en 15 días hábiles: Área geográfica, la forma de elaborar el producto y sus ingredientes, control de establecimientos y producción.

\section{Certificación}

La certificación autoriza al producto usar la palabra DENOMINACIÒN DE ORIGEN para la comercialización del producto, adquiriendo todos los beneficios que esto conlleva.

Se publica en la Gaceta Oficial del Estado, con un rango de 30 días para cualquier oposición. La Indicación Geográfica queda inscrita como marca por un lapso de 10 años a partir de la fecha de publicación. La inscripción puede ser renovada.

\section{Estándares de calidad}

Un producto con una DOP necesariamente tendrá que vincular estándares de calidad en sus fases de producción. Cualquier sistema de control de la calidad constituye un reto para este tipo de industrias (Dora et al., 2013). Una DOP, al estar conjuntamente enfocada en el trabajo comunitario, artesanal, empresarial e industrial, debe trabajar con una normativa fácil de aplicar, entender, seguir y controlar por parte del productor (Trienekens y Zuurbier, 2008).
De este modo él la cumplirá, y así se garantiza la seguridad del consumidor y mejora la competitividad de los productos (Cruz Gómez et al., 2004).

Una DOP se respaldará principalmente en la homologación de sus procesos productivos, con base en la legislación local y a los principios de calidad de cada país, considerando que la industria tiene que adaptarse a las necesidades del consumidor (Brissaud, Frein y Rocchi, 2013). Los organismos de control de calidad que se encargan de controlar las 
características de higiene, propiedades organolépticas, reproducibilidad de las características específicas del producto y trazabilidad de los alimentos con una DOP velarán por los estándares de calidad bajo especificaciones y características mínimas que llenen estos requerimientos (Curtis, 2005). Bajo es- tos principios una DOP se respaldará en un equilibrio entre la trazabilidad, seguridad alimentaria, reproducibilidad, características sensoriales y etiquetado, según se representa en el círculo de calidad propuesto en la Figura 1.

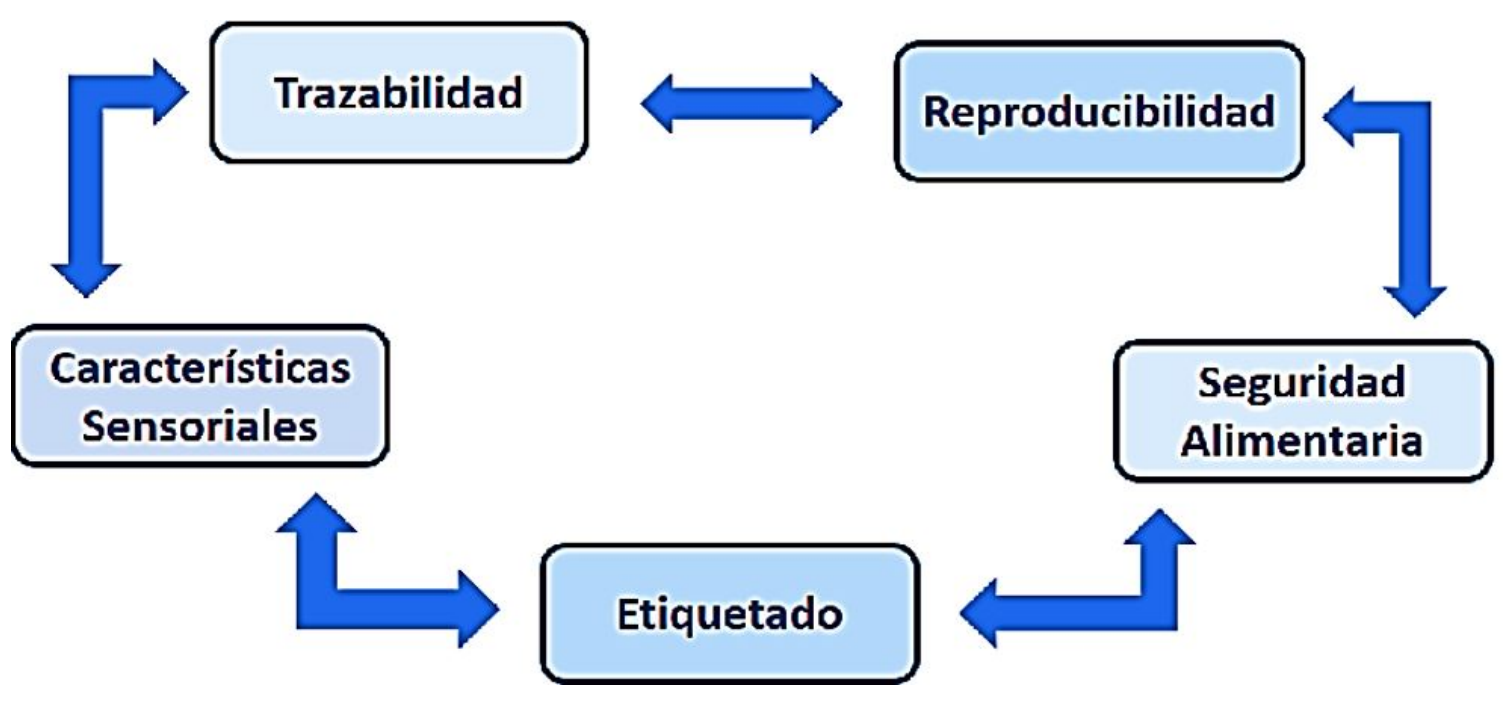

Figura 1. Círculo de la calidad.

\section{Definición de un pliego de condi- ciones para una DOP de Origen Animal}

Un pliego de condiciones consiste en una ficha de seguridad técnica donde se establecen los ingredientes, factores y condiciones para la implementación de una DOP, además de los causales de adulteración del mismo. Es recomendable que sea definida por los actores de la DOP, sea fácil de aplicar, entender, seguir y controlar (FAO, 2011). El pliego responde a las obligaciones legales de cada país, y se fundamenta en la investigación científica como base para el establecimiento de las normas de seguridad y calidad alimentaria (Vandecandelaere et al., 2010).

El manejo adecuado de la certificación y las condiciones que debe cumplir un producto con DOP, permite promocionar el producto globalmente, $\mathrm{y}$ conseguir un importante reconocimiento internacional, como está sucediendo con el Queso Manchego (Consejo Regulador Queso Manchego, 1995).

\section{Principios que vinculan una DOP de origen lácteo con su am- biente}

Un queso es la consecuencia de la combinación y correlación de varios factores, tanto intrínsecos como extrínsecos, que influyen en su composición y proceso de fabricación. Las correlaciones de estos factores afectan el rendimiento y composición quesera (Cecchinato y Bittante, 2016), siendo estos factores para tomar en cuenta en el pliego de condiciones de una DOP.

\section{Origen}

Un queso de calidad se definirá por el origen de su materia prima (especie, raza, lactancia, alimentación, CCS), que afecta la composición de la leche ( $\mathrm{pH}$, grasa, proteína), y específicamente la composición vinculada con las características del queso (caseína y grasa).

En los procesos industriales de fabricación de un queso, el resultado final dependerá del tratamiento térmico, cocción, uso de estárteres, aditivos, pun- 
tos de corte, sinéresis, desuerado, amasado, etc., entre muchos más factores que al ser modificados cambiarán notoriamente la calidad del queso (Fox et al., 2004). El desarrollo de sabores específicos en quesos, según el origen de la leche, se da principalmente por el aporte de bacterias no patógenas al queso (Yoon, Lee y Choi, 2016).

\section{Forma de Producción}

El proceso de producción de leche es un proceso complejo y debe ser controlado de acuerdo con los objetivos de cada ganadero. La forma de producir la materia prima influye en la cantidad y calidad de la leche, como sucede en Urgell (Consejo Regulador de la Mantequilla de Altura de Urgell, 2001), región donde se produce leche con niveles de grasa óptimos para la elaboración de mantequilla.

En el caso de la producción lechera en el Ecuador, en su mayoría proviene de sistemas extensivo que manejan parámetros productivos bajos. Requelme y Bonifaz (2012) indican que es necesario políticas dirigidas hacia los pequeños productores ya que estos son la mayoría y manejan producciones promedio inferiores a 4 litros diarios, en extensiones de 1 a 5 hectáreas. En este caso, una DOP se beneficiará de la especificidad de estas formas de producción y de allí obtendría su diferenciación frente a los demás productos.

La guía de Buenas Prácticas Pecuarias de Producción de Leche (AGROCALIDAD, 2012) abarca un amplio número de formas de producción a las que los distintos productores pueden adaptarse. $\mathrm{Pa}-$ ra el desarrollo de una DOP, esta guía es un punto de partida de la cual el productor puede garantizar la calidad de su materia prima.

\section{Alimentación de los animales}

La calidad de los productos lácteos depende, como ya se ha comentado, del origen de su materia prima, y ésta depende en buena medida del manejo y la alimentación de los rebaños. Así lo respaldan varios estudios que determinan que al alimentar los rebaños comerciales con pastos de diferentes tipos la composición de la leche varía, afectando a la vez las características finales de los quesos (Valdivielso et al., 2016).

Fabricación
La fabricación del queso afectará su calidad, y sus características sensoriales dependerán de varios factores (Johnson, 2017). Entre los aspectos sensoriales que se ven influenciados por la fabricación están: el carácter nutritivo, textura, sabor, aroma, y apariencia. También, la tecnología aportará aditivos y procesos operativos que pueden modificar el producto final. Durante la fabricación del queso existen otros factores, como el salado, moldeado, prensado, maduración, almacenamiento y empacado, que afectarán las características sensoriales del queso.

\section{Almacenamiento}

Dado que el queso se ve afectado por distintas variables desde su origen, su posterior almacenamiento también influirá en sus características organolépticas finales (Fox et al., 2004). Es así como las condiciones de humedad y temperatura afectarán directamente el producto como sucede en Cabrales, donde las condiciones de las cuevas en que maduran son especiales y tienen una micro biota específica para el propósito. Estas condiciones brindan las características únicas al queso ahí producido (Consejo Regulador del Queso Cabrales, 1990). Debido a que el consumidor ecuatoriano tiene entre sus preferencias quesillos, quesos frescos quesos amasados y quesos del tipo mozarela (NTE-INEN-1528, 2012) cuyo tiempo de conservación es corto el correcto almacenamiento y mantenimiento de la cadena de frío permitirá que el producto no pierda las características deseadas en el mercado.

\section{Ejemplo práctico de un pliego de condiciones aplicable al Ecua- dor}

Se debe tomar en consideración que entre los productos lácteos, el Ecuador se caracteriza por la comercialización de quesos frescos no curados, y que las regiones han desarrollado varios tipos de quesos cuya diferenciación radica en su distinto origen. Sobre la base de los pliegos de condiciones españoles (Consejo Regulador del Queso de Tetilla, 1983; Consejo Regulador de la Mantequilla de Altura de Urgell, 2001; Consejo Regulador Queso Cantabria, 2006; Consejo Regulador DOP Arzua y Ulloa, 2009; 
Consejo Regulador Queso de Murcia, 2011) se puede desarrollar un comparativo ejemplificado de los principios para la posible obtención de una DOP de origen animal en Ecuador, en particular para el Queso de Hoja de Cayambe, tal como se expone a continuación:

Nombre: Denominación de Origen Protegido "Queso de Hoja de Cayambe"

Descripción del Producto: Queso no madurado listo para el consumo directo, hecho a partir de cuajada de leche fresca, con masa acidificada, en presencia de bacterias mesófilas nativas no patógenas de Cayambe (NTE-INEN-1528, 2012), sometida a un incremento térmico previo al hilado y amasado del queso, y posteriormente envuelta en hojas de achira (Canna edulis) (Fairlie, Morales Bermúdez y Holle, 1999).

\section{Caracterización detallada de la materia prima}

Origen: Obtenido a partir de leche fresca de las fincas y haciendas de las parroquias del cantón Cayambe y Pedro Moncayo (Pichincha).

Características cualitativas: Hecho con leche de las siguientes características: densidad 1.028 a 1.034, extracto seco no graso $>8.2 \%$, proteína $>2.9 \%$, grasa $>3 \%$ y acidez $0.14 \%-0.18 \%$ (Banville et al., 2013).

Fases: La leche es recolectada diariamente en las fincas productores y almacenada a temperaturas $<7$ ${ }^{\circ} \mathrm{C}$ durante toda la cadena productiva.

Proceso: Leche tratada térmicamente $\left(66{ }^{\circ} \mathrm{C}, 30\right.$ minutos) y conservada a $<7^{\circ} \mathrm{C}(\mathrm{FAO}, 2011)$.

\section{Características y propiedades organolépticas del producto}

Organolépticas: (Incluye: pasta, textura, corteza, sabor, aroma). Queso de consistencia semi-blanda, de textura fibrosa y elástica, color blanco amarillento y uniforme, sabor láctico y dulce por la influencia de la hoja de achira, aroma poco desarrollado y láctico.

Físicas: (Incluye: forma, dimensión, peso). Consiste en una masa aplanada de queso hilado y enrollado sobre sí mismo, envuelto de una hoja de achira en forma de tamal, cada pieza tiene una dimensión de 8 a $15 \mathrm{~cm}$. y un peso de 25 a $100 \mathrm{~g}$.

Composición química: (Incluye: grasa, proteína, extracto seco, $\mathrm{pH})$. Con base en sus característi- cas, su composición posterior al desuerado tendrá una humedad de $46-48 \%$, concentración de proteína $18 \%$, y de grasa $20 \%$ (FUNIBER, 2016), y un pH al momento de empacar de 5.5 a 5.7 .

\section{Descripción detallada de la zona geográfica}

La descripción detallada de la zona geográfica debe incluir: regiones, división política, límites geográficos, mapas, etc. En este caso, Cayambe y Pedro Moncayo son cantones de Pichincha ubicados en la Sierra ecuatoriana.

El cantón Cayambe $\left(1382 \mathrm{~km}^{2}\right)$ se encuentra en el centro geográfico de la línea equinoccial $\mathrm{N}^{\circ} 0^{\prime}$. Limita al norte con los cantones Cotacachi, Otavalo y Pimampiro (Imbabura), al Sur con el cantón Quito (Pichincha) y el cantón Gonzalo Pizarro (Sucumbíos), al este con el Parque Nacional Cayambe-Coca, y al oeste el cantón Pedro Moncayo y Quito (Pichincha) (GADIP Cayambe, 2016).

El cantón Pedro Moncayo $\left(332 \mathrm{~km}^{2}\right)$ se ubica ligeramente al Norte de la línea equinoccial $\mathrm{N} 0^{\circ} 10^{\prime}$. Limita al Norte con el Cantón Cotacachi, al Sur con el Cantón Quito, al Este con el Cantón Cayambe, y al Oeste con el cantón Quito (GAD Pedro Moncayo, 2016).

\section{Elementos que prueban la procedencia del pro- ducto}

Seguimiento a las explotaciones ganaderas: Los procesos de producción son regulados por diferentes manuales de buenas prácticas ganaderas, como la Guía de Buenas Prácticas Pecuarias de Producción de Leche, Resolución Técnica n ${ }^{\circ} 217$ y n ${ }^{\circ} 842$, del 30 de noviembre del 2012 (AGROCALIDAD, 2012).

Seguimiento en las fases de producción: Los ganaderos y productores de materias primas de origen animal que piensan destinar sus productos para este propósito tendrán que basar sus prácticas en estas guías y ser auditados de forma conjunta frente a Agrocalidad (AGROCALIDAD, 2012).

Los productores de alimentos se basarán en la Norma Técnica de Buenas Prácticas de Manufactura para Alimentos Procesados en el Ecuador (Ministerio de Salud, 2015), y se apoyarán en normas afines y específicas como el Manual de Manufactura y Buenas Prácticas de la Elaboración de Productos Lácteos (FAO, 2011).

Control en el mercado: El control y manejo de la marca se basará en la Ley Orgánica de Regulación 
y Control del Poder del Mercado (Asamblea Nacional, 2011).

Control de las normas de calidad y Seguridad alimentaria: El control de las normas de calidad se hará con base en la norma NTE-INEN ISO 9001 (NTEINEN-ISO-9001, 2008), conforme a la NTE-INEN 2537 (NTE-INEN-2537, 2010).

Trazabilidad: De acuerdo con las políticas de autocontrol, se realizarán inspecciones internas que garanticen la trazabilidad con base en lo expuesto en las Guías de Buenas Prácticas Ganaderas.

\section{Vínculos con el medio}

Tradicional e históricos: Ubicado en las faldas del nevado, Cayambe es reconocida como una tierra de valles y estribaciones fértiles, en donde se ha desarrollado la agricultura durante generaciones. Considerada una zona tradicionalmente lechera, en esta ciudad se han instalado desde décadas atrás varios centros de procesamiento lácteo. A partir de su leche de "altura" (por encima de los $2000 \mathrm{msnm}$ ) se produce el "Queso de Hoja de Cayambe", siendo éste uno de los motores de la economía local junto a su tradicional bizcocho (GADIP Cayambe, 2016).

Social: La población de Cayambe y la nación Kayambi tienen un vínculo histórico precolombino con la región. Constituye una nación reconocida por el Consejo Nacional de Nacionalidades y Pueblos Indígenas, que impulsa activamente los procesos de desarrollo y reivindicación social que fomenta la Constitución de la República (Kayambi, 2016).

$\mathrm{Su}$ agrupación ha tenido gran influencia en diversos movimientos de lucha social, es una fuerza demográfica mayoritaria en las zonas de Cayambe y Pedro Moncayo en Pichincha, además de su participación social en provincias como Imbabura y Sucumbíos. Natural: Ubicado en la mitad geográfica del mundo. Cayambe tiene la particularidad de ser una región de clima templado y frío. Sus suelos son de origen volcánico y están rodeados por una orografía irregular, con alturas que oscilan entre los 1740 y $5245 \mathrm{msnm}$. La temperatura media regional no varía mayormente durante todo el año, fluctuando desde $11.8^{\circ} \mathrm{C}$ a $15.1^{\circ} \mathrm{C}$. La precipitación promedio anual varia de 730 a $940 \mathrm{~mm}$, según la región del cantón, con una disminución gradual de las mismas durante los meses de mayo a octubre (IEE, 2013). La vegetación de páramo rodea los valles y ocupan la sección inferior del piso alto andino de la Cordillera Central y del Nudo del Mojanda-Cajas
(GADIP Cayambe, 2016). En sus valles se encuentran los bosques alto montano y montano (Josee et al., 2009).

\section{Sistema de Producción}

Agrícola y ganadero: La producción ganadera en la región se hace con pastos mejorados. Principalmente se usan varias líneas de ryegrass (Lolium hybridum) y pastos nacionales asociados con la industria láctea (Lolium multiflorum var. Pichincha).

La línea ganadera se basa en vacunos mestizos y mejorados con líneas Holstein Frisona, así como con cruces con otras razas lecheras de origen europeo. La producción láctea se realiza principalmente en fincas de tamaño medio y pequeño (MenaVásconez, Boelens y Vos, 2016), mediante rebaños pequeños o comunitarios (Bonifaz García y de Jesús Requelme, 2011).

Manejo de la materia prima: El manejo del ganadero está enfocado en la alta calidad de pastos (Roca Fernández y González Rodríguez, 2014), y se basa en la capacidad agroecológica de la zona para la ganadería (Franzluebbers, Sawchik y Taboada, 2014).

Producción y transporte: La leche es ordeñada diariamente en las fincas o comunas del sector, y luego es llevada a las cubas de las fincas productoras o a los centros de acopio autorizados. Posteriormente es transportada directamente a los centros de elaboración artesanal o fábricas autorizadas de la ciudad de Cayambe.

Sistema de elaboración o industrialización: La leche es procesada de forma comunitaria o industrial (en fábricas autorizadas) y su producción es utilizada para la venta de queso de hoja en los comercios locales.

\section{Obtención del producto}

a) Se establece el "Queso de Hoja de Cayambe" basado en la clasificación de tipos de queso de la NTE-INEN-2537 (2010), numeral 2.1.17.

b) La coagulación de la leche se realizará entre 32 y $35^{\circ} \mathrm{C}$, durante 30 minutos.

c) Para permitir el desuerado se cortará la cuajada mediante cortes sucesivos. 
d) Se añadirá sal y ácido cítrico a la cuba dejándolo actuar sobre los granos.

e) Se recalentará la cuba superando los $60^{\circ} \mathrm{C}$.

f) Una vez que la masa esté suavizada, se empezará a amasar rápidamente procurando reducir las pérdidas de finos.

g) Se tomará la masa de queso hilado y se aplanará con un bolillo.

h) Se enrollará sobre sí mismo y se procederá a cortar.

i) Se seccionará la nervadura central de una hoja de achira para aplanarla y se la clarificará en agua hirviendo.

j) Se envolverá la pieza de queso con cada hoja y se empacará el producto inmediatamente.

\section{Comercialización y etiquetado}

El producto tiene que estar correctamente identificado, respetar los acuerdos comerciales y la normativa local establecida en la norma NTE 1334-1, 1334-2 y 1334-3 (NTE-INEN-1334, 2011). En ella se podrán identificar claramente los sellos reconocidos por el IEPI que lo cualifican como DOP, además de un logo que claramente vincule el producto con la zona geográfica y la marca colectiva. El queso debe expenderse en envases asépticos, herméticamente cerrados, que protejan el producto, sean resistentes, no alteren sus características organolépticas, y ayuden a su conservación y calidad. Además, deben asegurar su inocuidad durante el almacenamiento, transporte y expendio de acuerdo con la norma NTE 1528 (NTE-INEN-1528, 2012).

La comercialización del producto se debe hacer en centros de distribución y comercialización autorizados. Se debe definir una normativa que autorice la distribución de este queso, vinculándolo con la calidad de su procedencia.

\section{Estructura de control y registro}

Una vez autorizada la DOP, en este apartado se incluirá la autorización otorgada por el IEPI de acuerdo con el marco legal ya establecido. Constarán las direcciones, contactos y medios digitales de la DOP, así como los derechos y obligaciones de los actores de la DOP, la lista de productores, manufacturadores y comercializadores autorizados, junto a las respectivas acreditaciones.

\section{Conclusiones}

Una DOP legalmente constituida es reconocida en todo el mundo, y por ello es necesario armonizar la legislación ecuatoriana con respecto a otros estados latinoamericanos y la propia legislación europea. Este tipo de estudios ayudan a plantearse las bases para el desarrollo de una ley específica para una DOP. Para poder desarrollar una DOP es necesario crear una normativa que se adapte a la coyuntura de cada país. En el Ecuador es posible establecer una DOP siempre y cuando exista flexibilidad suficiente que permita la vinculación de varios actores para su desarrollo. Para que las DOP resulten comercialmente atractivas es necesario impulsar la investigación y fomentar la inversión productiva. Para ello es necesario profundizar los estudios multidisciplinarios en el aspecto legal, estudios de calidad interna, buenas prácticas de manufactura, e impacto económico para su desarrollo. Debido a que nacen en muchas ocasiones de la voluntad de la propia comunidad, las DOP pueden ser impulsoras socio-económicas rurales en la región, sobre otro tipo de programas de cooperación internacional e iniciativas productivas industriales. Por el contrario, las políticas públicas, la poca capacidad asociativa y la baja calidad de los productos, limitan el éxito de una DOP. Frente a la coyuntura global de libre comercio interregional, el Ecuador tiene que estar preparado para el uso de alternativas productivas. Con base en el ejemplo del pliego de condiciones del "Queso de Hoja de Cayambe", desarrollado en este trabajo, se puede validar esta propuesta in situ de acuerdo con las características del producto ampliando su alcance e impacto a otras regiones del país.

\section{Agradecimientos}

Al SENESCYT y su programa de Convocatorias Abiertas 2014.

\section{Referencias}

ADPIC. 1994. “Acuerdo de la Ronda de Uruguay; Parte II, Normas relativas a la existencia, alcance y ejercicio de los derechos de propiedad intelectual. Art 22 y 23." Acuerdo de la Ronda de Uruguay. [en línea] disponible: https://goo.gl/ UBGiPp. 
AGROCALIDAD. 2012. "Buenas Prácticas Pecuarias en la Producción de Leche." Agencia Ecuatoriana de Aseguramiento de la Calidad del Agro. [en línea] disponible en: https://goo.gl/xZn1x6.

Asamblea Constituyente. 2008. "Constitución de la República del Ecuador." [en línea] disponible: https://goo.gl/1ehukU.

Asamblea Nacional. 2011. “Ley Orgánica de Regulación y Control del Poder del Mercado." Ecuador. [en línea] disponible: https:/ /goo.gl/ePPJiZ.

Asamblea Nacional. 2016. “Código Orgánico de la Economía Social de los Conocimientos, Creatividad e Innovación, (Código Ingenios). Capítulo XII." Ecuador. [en línea] disponible: https: //goo.gl/94oRdt.

Banville, V., P. Morin, Y. Pouliot y M. Britten. 2013. "Physical properties of pizza Mozzarella cheese manufactured under different cheese-making conditions." Journal of Dairy Science 96(8):48044815. [en línea] doi: https://doi.org/10.3168/jds. 2012--6314.

Bonifaz García, N y N. de Jesús Requelme. 2011. "Buenas prácticas de ordeño y la calidad higiénica de la leche en el Ecuador." La Granja Revista de Ciencias de la Vida 14(2):45-57. [en línea] doi: http:/ /dx.doi.org/10.17163/lgr.n14.2011.04.

Brissaud, D, Y Frein y V. Rocchi. 2013. "What Tracks for Sustainable Production Systems in Europe?" Procedia CIRP 7:9-6. [en línea] doi: https: //doi.org/10.1016/j.procir.2013.05.003. Forty Sixth CIRP Conference on Manufacturing Systems 2013.

Café de Colombia. 2009. "IGP/DO." Federación Nacional de Cafeteros de Colombia. [en línea] disponible: https://goo.gl/Zy3Up8.

Cecchinato, A. y G. Bittante. 2016. "Genetic and environmental relationships of different measures of individual cheese yield and curd nutrients recovery with coagulation properties of bovine milk." Journal of Dairy Science 99(3):19751989. [en línea] doi: https://doi.org/10.3168/jds. 2015--9629.

Comisión de la Comunidad Andina. 2000. "Decisión 486 de la Comunidad Andina. Título XII, Artículos 201 al 223." [en línea] disponible: https: //goo.gl/JY6RN3.
Consejo de la Unión Europea. 2006. "Reglamento (CE) No 510/2006 del Consejo sobre la protección de las indicaciones geográficas y de las denominaciones de origen de los productos agrícolas y alimenticios 2 de Julio 2008." [en línea] disponible: https://goo.gl/2GRgMV.

Consejo de la Unión Europea. 2012. "Reglamento (UE) No 1151/2012 del Parlamento Europeo y del Consejo de 21 de noviembre de 2012 sobre los regímenes de calidad de los productos agrícolas y alimenticios." [en línea] disponible: https://goo.gl/A4hqcy.

Consejo Regulador de la Mantequilla de Altura de Urgell. 2001. "Pliego de Condiciones de la Denominación de Origen Protegida "Mantequilla de l'Alt Urgell y la Cerdanya" o "Mantega de l'alt Urgell i la Cerdanya"." [en línea] disponible: https://goo.gl/jeM2nb.

Consejo Regulador del Queso Cabrales. 1990. “Denominación de Origen Protegida "Queso Cabrales" Pliego de Condiciones." [en línea] disponible: https://goo.gl/RGZ7ub.

Consejo Regulador del Queso de Tetilla. 1983. “Expediente de la Denominación de Origen Queso de Tetilla Galicia." [en línea] disponible: https: //goo.gl/XcCfjP.

Consejo Regulador DOP Arzua y Ulloa. 2009. “Expediente de la Denominación de Origen Protegida (DOP) "Arzúa-Ulloa"." [en línea] disponible: https://goo.gl/W6gS7Z.

Consejo Regulador Queso Cantabria. 2006. "Reglamento de la Denominación de Origen "Queso de Cantabria"." [en línea] disponible: https: //goo.gl/cJUxSN.

Consejo Regulador Queso de Murcia. 2011. “Expediente de la Denominación de Origen Protegida (DOP) Queso de Murcia." [en línea] disponible: https://goo.gl/iHUXTE.

Consejo Regulador Queso Manchego. 1995. “Denominación de Origen Protegida "Queso Manchego" Pliego de Condiciones." [en línea] disponible: https://goo.gl/H7dk1k.

Cruz Gómez, J. C, B Lucena Cobos, M Méndez Rodríguez y F. Cáceres Clavero. 2004. "Sistemas de certificación de la calidad en el sector agroalimentario español." Unidad de Prospectiva de la 
Consejería de Agricultura y Pesca de la Junta de Andalucía. [en línea] disponible: https:/ /goo.gl/ gzoyEM.

Dora, Manoj, Maneesh Kumar, Dirk Van Goubergen, Adrienn Molnar y Xavier Gellynck. 2013. "Food quality management system: Reviewing assessment strategies and a feasibility study for European food small and medium-sized enterprises." Food Control 31(2):607-616. [en línea] doi: https://doi.org/10.1016/j.foodcont.2012.12.006.

Fairlie, T, M Morales Bermúdez y M. Holle. 1999. Raíces y tubérculos andinos. Avances de Investigacíon. INIAP-Ecuador. [en línea] disponible: https: //goo.gl/Dhuj8T.

FAO. 2011. "Procesos para la elaboración de productos lácteos." Organización de las Naciones Unidas para la Alimentación y la Agricultura. [en línea] disponible: https://goo.gl/XdKvuC.

Fox, P, P McSweeney, T Cogan y TGuinee . 2004. Cheese Chemistry Physics and Microbiology: Factors That affect Quality of Cheese ( $p p$. 582-587). Elsevier Academic Press. [en línea] disponible: https: //goo.gl/2tGMg9.

Franzluebbers, A. J, J Sawchik y M. Taboada. 2014. "Agronomic and environmental impacts of pasture-crop rotations in temperate North and South America." Agriculture, Ecosystems \& Environment 190:18-26. [en línea] doi: https://doi. org/10.1016/j.agee.2013.09.017. Integrated CropLivestock System Impacts on Environmental Processes.

GAD Pedro Moncayo. 2016. “Gobierno Autónomo Descentralizado del Cantón Pedro Moncayo." [en línea] disponible: https:/ /goo.gl/zRu1Da.

GADIP Cayambe. 2016. "Municipio del Cantón Cayambe." [en línea] disponible: https://goo.gl/ anmtJk.

Guardia, M. D. 2016. "Memorias de Charla Magistral Máster de Calidad de Alimentos de Origen Animal. IRTA." Universidad Autónoma de Barcelona.

Guerrero, Luis, Anna Claret, Wim Verbeke, Geraldine Enderli, Sylwia Zakowska-Biemans, Filiep Vanhonacker, Sylvie Issanchou, Marta Sajdakowska, Britt Signe Granli, Luisa Scalvedi, Mi- chele Contel y Margrethe Hersleth. 2010. "Perception of traditional food products in six European regions using free word association." Food Quality and Preference 21(2):225-233. [en línea] doi: https://doi.org/10.1016/j.foodqual.2009.06. 003. Third European Conference on Sensory and Consumer Research: A Sense of Innovation.

Hentschel, Jesko y William F Waters. 2002. “Rural Poverty in Ecuador: Assessing Local Realities for the Development of Anti-poverty Programs." World Development 30(1):33-47. [en línea] doi: https://doi.org/10.1016/S0305--750X(01) 00099--7.

IEE. 2013. "Memoria Técnica de Cantón Cayambe: Generación de Geo información para la Gestión del Territorio a Nivel Nacional. Clima e Hidrología." Instituto Espacial Ecuatoriano, Ministerio de Defensa, Secretaría Nacional de Planificación y Desarrollo, Gobierno de Pichincha. [en línea] disponible: https://goo.gl/k1GjiA.

IEPI. 2016. "Denominación de Origen Identidad Ecuatoriana en Productos Propios." Instituto Ecuatoriano de la Propiedad Intelectual. [en línea] disponible: https://goo.gl/KwFXtW.

Johnson, M.E. 2017. "A 100-Year Review: Cheese production and quality." Journal of Dairy Science 100(12):9952-9965. [en línea] doi: https:/ / doi. org/10.3168/jds.2017--12979.

Josee, C, F Cuesta, G Navarro, V Barrena, E Cabrera, E Chacón-Moreno, W Ferreira, M Peralvo, J Saito y A. Tovar. 2009. "Ecosistemas de los Andes del Norte y Centro. Bolivia, Colombia, Ecuador, Perú y Venezuela. pp. 20-24." Secretaría General de la Comunidad Andina, Programa Regional ECOBONA-Intercooperation, CONDESANProyecto Páramo Andino, Programa BioAndes, EcoCiencia, NatureServe, IAVH, LTA-UNALM, ICAE-ULA, CDC-UNALM, RUMBOL SRL. Lima. [en línea] disponible: https://goo.gl/5oWDeA.

Kayambi. 2016. "Reseña histórica de los Kayambis." [en línea] disponible: https:/ /goo.gl/2eVahy.

Legislación Chilena. 2005. “Ley Número 19039, Ley de Propiedad Industrial Titulo IX." Ministerio de Economía, Fomento y Reconstrucción. [en línea] disponible: https://goo.gl/GJn5xZ. 
Llorente, M. 2001. “La fuerza de la diferencia. La Denominación de Origen un instrumento, para el desarrollo." La Val de O, Huesca España. [en línea] disponible: https://goo.gl/vAaXGq.

Maldonado, M. 2017. “Estudio comparativo para obtención de una D.O.P. en Ecuador en base al caso europeo." CGSIN 2CNIA-2017-032. [en línea] disponible en: https://goo.gl/i4RufN.

Mena-Vásconez, Patricio, Rutgerd Boelens y Jeroen Vos. 2016. "Food or flowers? Contested transformations of community food security and water use priorities under new legal and market regimes in Ecuador's highlands." Journal of Rural Studies 44:227-238. [en línea] doi: https://doi.org/ 10.1016/j.jrurstud.2016.02.011.

Ministerio de Salud. 2015. "Norma Técnica Sustitutiva de Buenas Prácticas de Manufactura para Alimentos Procesados en Ecuador. Registro Oficial 555." Ecuador. [en línea] disponible: https: //goo.gl/LHBQQ6.

Molina, M. 2015. "La protección de la Denominación de Origen, indicación geográfica e indicación de procedencia. estudio comparativo de las legislaciones de los estados latinoamericanos y español." RIVAR 2(6):46-69. [en línea] disponible: https://goo.gl/2BUZFj.

NTE-INEN-1334. 2011. "Rotulado de Productos Alimenticios para Consumo Humano.".

NTE-INEN-1528. 2012. "Norma General para Quesos no Madurados.".

NTE-INEN-2537. 2010. "Sistema de Gestión Integral para la Micro, Pequeña y Mediana Empresa. Requisitos.".

NTE-INEN-ISO-9001. 2008. "Sistemas de Gestión de Calidad.".

Oberthür, Thomas, Peter Läderach, Huver Posada, Myles J. Fisher, Luis F. Samper, Julia Illera, Laure Collet, Edgar Moreno, Rodrigo Alarcón, Andres Villegas, Herman Usma, Carolina Perez y Andy Jarvis. 2011. "Regional relationships between inherent coffee quality and growing environment for denomination of origin labels in Nariño and Cauca, Colombia." Food Policy 36(6):783-794. [en línea] doi: https://doi.org/10. 1016/j.foodpol.2011.07.005. Between the Global and the Local, the Material and the Normative: Power struggles in India's Agrifood System.

Oyarzun, M. T. 2011. "Memorias del SeminarioTaller de las Indicaciones Geográficas y Denominaciones de Origen en Ecuador." Organización de las Naciones Unidas para la Alimentación y la Agricultura. [en línea] disponible: https:/ / goo. gl/Y9eEv6.

Requelme, N y N. Bonifaz. 2012. "Caracterización de Sistemas de Producción Lechera de Ecuador." La Granja Revista de Ciencias de la Vida 12(1):5569. [en línea] doi: http://dx.doi.org/10.17163/ lgr.n15.2012.05 .

Roca Fernández, A. I y A. González Rodríguez. 2014. "Ingestión de hierba y producción de leche en pastoreo." Informativo Veterinario p. [en línea] disponible: https://goo.gl/3r1aMQ.

Sellers, R y J. Nicolau. 2001. "La calidad y su impacto sobre la rentabilidad y la volatilidad." Universidad de Alicante. Departamento de Marketing. [en línea] disponible: https://goo.gl/19t5xe.

Senado Argentino. 2004. "Ley Argentina, Ley 25.380, Régimen Legal para las Indicaciones de Procedencia y Denominaciones de Origen de Productos Agrícolas y Alimentarios." [en línea] disponible: https://goo.gl/gA1yTb.

Tolentino Martínez, J. M. 2015. La Denominación de Origen como elemento de análisis institucional. El caso del arroz de Morelos. In 20 Encuentro Nacional Sobre Desarrollo Regional en México. Cuernavaca, Morelos Amecdir UNA. [en línea] disponible: https://goo.gl/AinWJC.

Trienekens, Jacques y Peter Zuurbier. 2008. “Quality and safety standards in the food industry, developments and challenges." International Journal of Production Economics 113(1):107-122. [en línea] doi: https://doi.org/10.1016/j.ijpe.2007.02. 050. Research and Applications in E-Commerce and Third-Party Logistics Management Special Section on Meta-standards in Operations Management: Cross-disciplinary perspectives.

Unión de Lisboa. 1979. “Arreglo de Lisboa relativo a la Protección de las Denominaciones de Origen y su Registro Internacional del 31 de octubre de 1958, revisado en Estocolmo el 14 de julio de 1967 y modificado el 28 de septiembre de 1979." [en línea] disponible: https://goo.gl/CKspPm. 
Unión Europea. 2014. "Comisión Europea Seguridad Alimentaria; De la granja a la mesa: alimentos sanos y seguros para todos." [en línea] disponible: https://goo.gl/2Yf545.

Unión Europea. 2015. “Geneva Act of the Lisbon Agreement on Appellations of Origin and Geographical Indications. Chp III y IV." [en línea] disponible: https://goo.gl/QZzjaJ.

Valdivielso, I., M.A. Bustamante, A. Aldezabal, G. Amores, M. Virto, J.C. Ruiz de Gordoa, M. de Renobales y L.J.R. Barron. 2016. “Case study of a commercial sheep flock under extensive mountain grazing: Pasture derived lipid compounds in milk and cheese." Food Chemistry 197:622-633. [en línea] doi: https://doi.org/10.1016/j.foodchem. 2015.10.133.

Vandecandelaere, E, F Arfini, G Belletti y A. Mares- cotti. 2010. "Uniendo personas, territorios y productos, guía para fomentar la calidad vinculada al origen y las indicaciones geográficas sostenibles." Organización de las Naciones Unidas para la Alimentación. [en línea] disponible: https: //goo.gl/Bs1U6o.

WTO. 2009. "TPR 214 Informe de políticas y prácticas comerciales." [en línea] disponible: https:/ / goo.gl/zGXQda.

WTO. 2012. "Revista General de la Evolución del Entorno Comercial Internacional." [en línea] disponible: https://goo.gl/1S297n.

Yoon, Yohan, Soomin Lee y Kyoung-Hee Choi. 2016. "Microbial benefits and risks of raw milk cheese." Food Control 63:201-215. [en línea] doi: https: //doi.org/10.1016/j.foodcont.2015.11.013. 